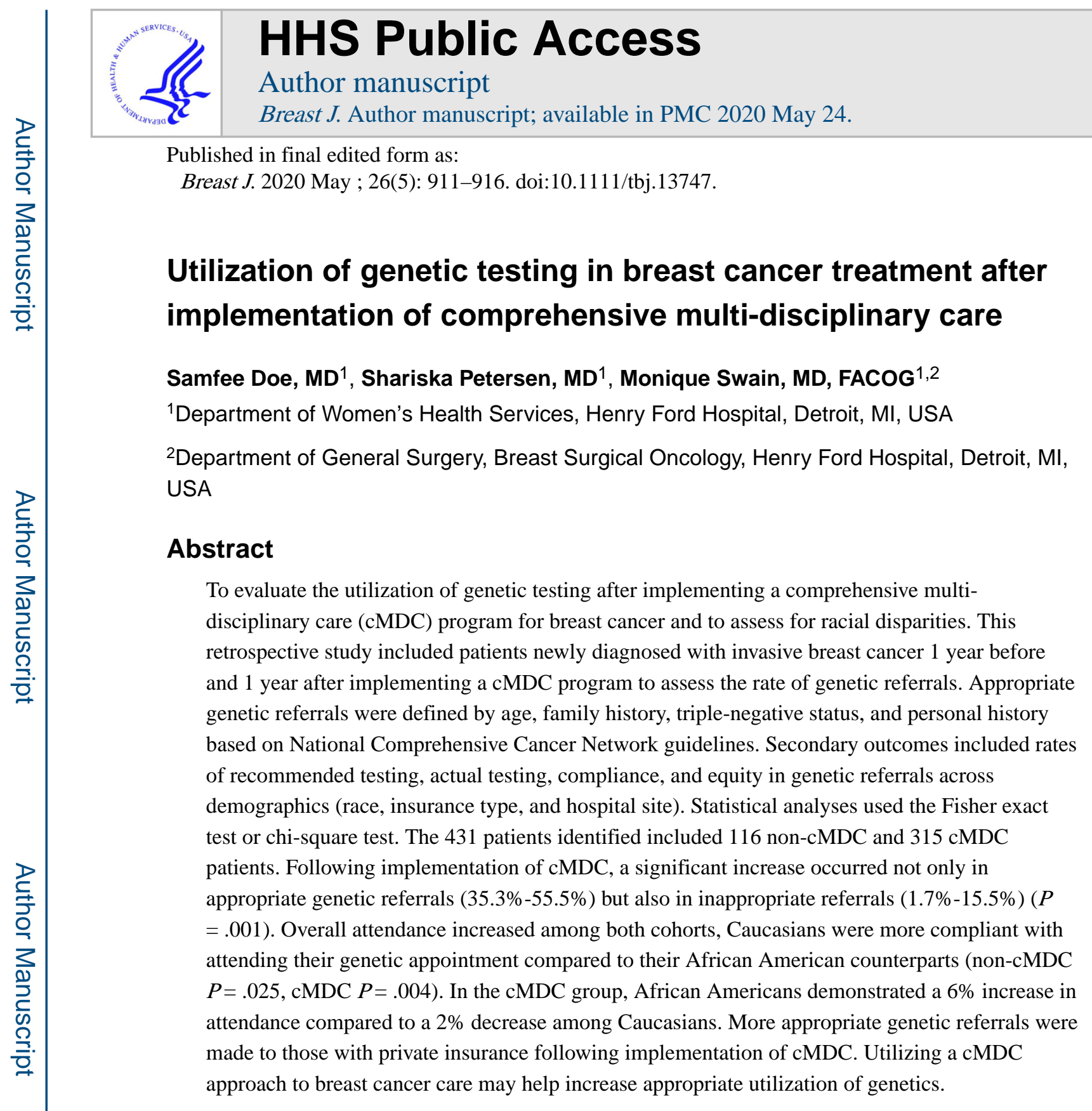

Keywords

compliance; genetic testing; multi-disciplinary cancer care program; racial disparities

\title{
1I INTRODUCTION
}

Historically, surgery was the primary method for treating breast cancer. ${ }^{1}$ More recently, the diagnosis and treatment of breast malignancies have escalated in their sophistication and complexity. ${ }^{2,3}$ With the advent of screening mammography, hormonal therapy, chemotherapy, and breast-conservation treatment, medical and radiation oncologists now have major roles in curative treatment. Therefore, the direction of breast cancer care has

Correspondence Monique Swain, Henry Ford Health System, Women's Health Services-Breast diseases, New Center One 8th Floor, 3031 W. Grand Blvd, Detroit, MI 48202, USA. MSwain1@hfhs.org. 
moved toward a multifaceted approach with the involvement of many disciplines of medicine, both diagnostic and therapeutic. ${ }^{4-6}$

One key player in the multi-disciplinary breast tumor board should be the geneticist as genetic screening is an integral part of breast cancer treatment. ${ }^{7-10}$ The identification of the BRCA gene offers an unprecedented opportunity for high-risk members of families with hereditary breast-ovarian cancer to learn whether they carry a cancer-predisposing mutation.

${ }^{11}$ Women found to carry a BRCA mutation have up to an $85 \%$ lifetime risk of breast cancer, a 20\%-65\% lifetime risk of ovarian cancer, and an increased risk of colon cancer. ${ }^{12}$ Men who carry the BRCA mutation are at increased risk for prostate and colon cancer and can also transmit the gene mutation to their daughters. Early identification of BRCA mutation carriers within hereditary breast-ovarian cancer families can allow for targeted surveillance and management strategies. ${ }^{13,14}$

Fewer than 1 in 5 individuals with breast cancer who meet National Comprehensive Cancer Network (NCCN) criteria have undergone genetic testing. ${ }^{15}$ Rates of appropriate referrals for genetic testing have been reported to be higher in a multi-disciplinary setting. ${ }^{16}$ Evidence also suggests that racial disparities exist with genetic referrals in patients with cancer. ${ }^{17,18}$ Our academic health system in southeast Michigan recently adopted a comprehensive and structured multi-disciplinary care approach to breast cancer treatment, including the participation of a geneticist. This study aimed to evaluate the appropriate referral and use of genetic testing after implementing a comprehensive multi-disciplinary care (cMDC) program and to assess for differences by race, location of care, and insurance status.

\section{I MATERIALS AND METHODS}

This retrospective study was approved with waiver of consent by the health system's institutional review board. We used the electronic medical record system to identify all patients from February 2015 to February 2017 with newly diagnosed invasive breast cancer who were treated at two of the health system sites, one suburban and one urban location. The cMDC program at both sites involved the same staff but was held on different days of the week. Patients who received treatment at outside institutions or other locations of our health system were excluded. The primary outcome was to assess the impact of the cMDC program on appropriate genetic referral, and secondary outcomes were to assess any differences between the two sites of care, insurance status, and race in appropriate utilization of genetic services.

Patients were categorized as non-cMDC (February 2015 to January 2016) and cMDC (February 2016 to February 2017). Data obtained from chart review included age, race, parity, county of residence, gender, and type of insurance (private vs public). Patients who had Medicare and private insurance were categorized as having private insurance. Genetic testing referrals were categorized as appropriate based on NCCN guidelines ${ }^{19}$ :

1. early age onset breast cancer

2. two breast primaries or breast and ovarian cancer in a single individual 
3. Two or more breast primaries or breast and ovarian cancers in close relatives from the same side of family

4. clustering of breast cancer with one or more of the following: thyroid cancer, sarcoma, adrenocortical carcinoma, endometrial cancer, pancreatic cancer, brain tumors, dermatologic manifestations or leukemia/lymphoma on the same side of the family

5. member of a family with a known mutation in a breast cancer susceptibility gene

6. population at risk ie women of Ashkenazi Jewish descent with breast or ovarian cancer at any age

7. any male breast cancer

8. ovarian cancer on the same side of family.

The geneticist was present at all tumor board discussions. When a patient met the NCCN criteria, a referral to the geneticist was placed, and the patient was scheduled to meet with a genetic counselor the same day after tumor board.

\subsection{Statistical analysis}

Sample size calculation, using a power of 0.80 , national rate of appropriate genetic referral rate of $20 \%$ and a type I error rate of 5\%, determined a sample size of 246 was needed for an adequately powered study. Statistical analyses used Fisher exact test in the presence of sparse categories (ie, expected cell counts $<5$ ) or chi-square test. All analyses were done with SPSS Statistics (IBM), with significance set at $P<.05$.

\section{I RESULTS}

The 431 patients identified included 116 (26.9\%) non-cMDC and 315 (73.1\%) cMDC patients (Table 1). The average age was 61 years, and $43.4 \%$ were African American (AA) and $56.4 \%$ Caucasian (CA). More patients had private insurance (65.7\%) than public insurance (33.9\%). The cMDC and non-cMDC groups were similar in demographics, except more patients were seen at the urban location. The overall rate of genetic referrals was higher after implementation of cMDC $(37 \%-71 \%, P=.001)$. However, the cMDC group also demonstrated a higher rate of inappropriate referrals $(15.5 \%$ vs $1.7 \%, P=.001)$ (Table $1)$.

Genetic referral overall increased for both races following implementation of cMDC (35.7\%-67.2\% AA, $P=.001$, and 38.3\%-73.8\% CA, $P=.001$ ). Within each cohort, AA and CA patients were offered genetic appointments at similar rates (Table 2). Overall, AA patients were less likely to comply with attending the genetics appointment (86\% AA vs $98 \%$ CA for cMDC, $P=.004$, and $80 \%$ AA vs $100 \%$ CA for non-cMDC, $P=.025$; Table 2 ). When patients attended the genetics appointment, genetic testing was recommended at similar rates for AA and CA patients (Table 2). For those whom testing was recommended, rates of actual testing were similar for both races (91\% vs $92 \%$ non-cMDC, $P=.968$, and $98 \%$ vs $100 \%$ cMDC, $P=.337$ ). 
In the cMDC group, appropriate genetic referrals were slightly higher at the suburban than the urban location, but not statistically significant $(60.7 \%$ vs $50.9 \%, P=.082$; Table 3$)$. Inappropriate referrals were offered similarly at both locations. Significantly more cMDC patients attended their appointment at the suburban location vs the urban location (97.6\% vs $89.3 \%, P=.047)$. Genetic testing was recommended at similar rates at both locations (Table 3 ). For those whom testing was recommended, rates of actual testing were similar across locations $(90.5 \%$ vs $93 \%, P=.802$, and $98 \%$ vs $100 \%, P=.242)$.

Genetic referral was offered (both appropriately and inappropriately) more often in cMDC patients with private than public insurance $(76.7 \%$ vs $60.8 \%, P=.003$; Table 4$)$. Although inappropriate genetic referrals were made similarly in both cohorts, more patients with private insurance received appropriate referrals than those with public insurance $(61.2 \%$ vs $44.9 \%, P=.006)$. Genetic appointments were attended at similar rates, and genetic testing was recommended at similar rates across cohorts (Table 4). For those whom testing was recommended, rates of actual testing were similar across insurance types (100\% vs $88 \%, P$ $=.251$, and $100 \%$ vs $97 \%, P=.303)$.

Overall, once referred, genetic testing was recommended or offered at a rate of $88.8 \%$ and performed at a rate of $95.8 \%$. No statistical difference was found between study groups.

\section{I DISCUSSION}

This study showed a significant increase in genetic referrals as well as scheduled and attended genetic appointments following implementation of cMDC for patients with invasive breast cancer at our institution. This may be attributed to scheduling an appointment at the time of tumor board instead of addressing it at a later time. Our study findings are consistent with the literature that indicates genetic referrals have been underutilized for eligible women based on NCCN guidelines. ${ }^{20,21}$

The literature also suggests that a lower proportion of AA women undergo genetic testing raising concerns about racial disparities in genetic counseling and testing. ${ }^{22,23}$ The overall rate of genetic referral increased with the implementation of cMDC and did not disproportionately increase based on race. However, AA women were less likely to attend their genetic appointments. Among the women who did attend their appointments, there was no difference in the rates of genetic testing recommended or completed, suggesting that the barrier to testing most likely lies in the adherence to genetic appointments in this cohort.

Although genetic referrals increased following the implementation of cMDC, the rates of genetic testing recommended and subsequently performed by the geneticist were similar between the study groups. This suggests that lack of referral for eligible patients in the noncMDC group may have been the limiting factor for receipt of the service, as genetic services were provided once referred. The number of inappropriate referrals also increased with the implementation of $\mathrm{cMDC}$, which indicates providers may require reminders or education about NCCN criteria, especially as inappropriate referral may be associated with unnecessary health care costs. A cost-benefit analysis was not a goal of this study, but this 
finding highlights the need for providers to adhere to NCCN guidelines for genetic referral in the management of patients diagnosed with invasive breast disease.

While genetic appointments were offered significantly more at both the urban and suburban location following the implementation of cMDC, genetic appointments were attended more often at the suburban location. Several factors have been associated with offering genetic referral, including college education, age below 45 years, household income $>\$ 35000$ in the year prior to diagnosis, and private health insurance. ${ }^{24-26}$ In our study, genetic referrals were more likely to be offered to patients with private insurance. An association between location of facility and genetic referral has not been previously reported, and future studies are needed to evaluate the observed difference in this study population.

Limitations of this study include its retrospective design, and thus, findings can only be associated with, not causative of, implementation of cMDC. Our study population may not be generalizable to other groups across the United States.

\section{I CONCLUSION}

Implementation of a cMDC approach to invasive breast cancer care at our academic health system significantly improved rates of appropriate patient referral to and patient use of genetic services regardless of race, location of care, or type of insurance. As a testament to the overall success of the program, referral to genetic screening services improved to $71 \%$ following implementation and subsequent genetic testing was recommended or offered at an astounding rate of $88.8 \%$ and performed at a rate of $95.8 \%$, compared to the $20 \%$ national rate of genetic testing. While no differences were found between AA and CA patients in referral to genetic services or in recommendation for genetic testing, more AAs did not attend their genetic appointment compared to CAs both before and after implementation of cMDC. This suggests more effort is needed to identify reasons for missed appointments to overcome this disparity. However, we must note that there was an overall high rate $(>80 \%)$ of genetic appointment attendance in both cohorts. Our study also suggests that providers may require reminders or education to follow the NCCN guidelines as inappropriate referrals for genetic services significantly increased after cMDC implementation.

\section{ACKNOWLEDGEMENTS}

The authors would like to thank Mary Quigg, MD, Myah Bell, MD, Vini Chopra, MD, and Lisa Newman, MD, for support formulating and collecting data for this project as well as Gordon Jacobsen, MS, for the statistical support for this manuscript.

\section{REFERENCES}

1. Halsted WSI. The results of radical operations for the cure of carcinoma of the breast. Ann Surg. 1907;46:1-19.

2. Fisher B, Fisher ER. The interrelationship of hematogenous and lymphatic tumor cell dissemination. Surg Gynecol Obstet. 1966;122:791-798. [PubMed: 5934190]

3. Rabinowitz B Interdisciplinary breast cancer care: declaring and improving the standard. Oncology. 2004;18:1263-1268; discussion 1268-1270, 1275. [PubMed: 15526830]

4. Tripathy D Multidisciplinary care for breast cancer: barriers and solutions. Breast J. 2003;9:60-63. [PubMed: 12558678] 
5. Shuster TD,Girshovich L, Whitney TM, Hughes KS. Multidisciplinary care for patients with breast cancer. Surg Clin North Am. 2000;80:505-533. [PubMed: 10836005]

6. Kaufman CS. Breast care is a team sport. Breast J. 2004;10:469-472. [PubMed: 15327510]

7. Easton DF, Pharoah PDP, Antoniou AC, et al. Gene-panel sequencing and the prediction of breastcancer risk. N Engl J Med. 2015;372:2243-2257. [PubMed: 26014596]

8. Mavaddat N, Pharoah PDP, Michailidou K, et al. Prediction of breast cancer risk based on profiling with common genetic variants. J Natl Cancer Inst. 2015;107:djv036. [PubMed: 25855707]

9. Hortobagyi GN, Chen D, Piccart M, et al. Correlative analysis of genetic alterations and everolimus benefit in hormone receptor-positive, human epidermal growth factor receptor 2-negative advanced breast cancer: results from BOLERO-2. J Clin Oncol. 2016;34:419-426. [PubMed: 26503204]

10. Daly MB, Pilarski R, Axilbund JE, et al. Genetic/familial high-risk assessment: breast and ovarian, version 2.2015. J Natl Compr Canc Netw. 2016;14:153-162. [PubMed: 26850485]

11. Du H, Davis A, Gao R, et al. Abstract 2192: Single-molecule single-cell DNA sequencing identifies ongoing copy number evolution in BRCA breast cancers [abstract]. Cancer Res. 2018;78:2192.

12. Tung N, Battelli C, Allen B, et al. Frequency of mutations in individuals with breast cancer referred for BRCA1 and BRCA2 testing using next-generation sequencing with a 25-gene panel. Cancer. 2015;121:25-33. [PubMed: 25186627]

13. Burstein HJ, Lacchetti C, Griggs JJ. Adjuvant endocrine therapy for women with hormone receptor-positive breast cancer: American Society of Clinical Oncology clinical practice guideline update on ovarian suppression summary. J Oncol Pract. 2016;12:390-393. [PubMed: 26931400]

14. Coates AS, Winer EP, Goldhirsch A, et al. Tailoring therapies-improving the management of early breast cancer: St Gallen International Expert Consensus on the Primary Therapy of Early Breast Cancer 2015. Ann Oncol. 2015;26:1533-1546. [PubMed: 25939896]

15. Childers CP, Childers KK, Maggard-Gibbons M, Macinko J. National estimates of genetic testing in women with a history of breast or ovarian cancer. J Clin Oncol. 2017;35:3800-3806. [PubMed: 28820644]

16. Gomez CL, Dawson NA, Dvorak RL, et al. Rates of appropriate genetic testing referral for breast cancer patients treated in a multi-disciplinary cancer care setting [abstract]. J Clin Oncol. 2014;32:45.

17. Manrriquez E, Chapman JS, Mak J, Blanco AM, Chen LM. Disparities in genetics assessment for women with ovarian cancer: can we do better? Gynecol Oncol. 2018;149:84-88. [PubMed: 29605055]

18. Halbert CH, Kessler L, Stopfer JE, Domchek S, Wileyto EP. Low rates of acceptance of BRCA1 and BRCA2 test results among African American women at increased risk for hereditary breastovarian cancer. Genet Med. 2006;8:576-582. [PubMed: 16980814]

19. Gradishar WJ, Anderson BO, Balassanian R, et al. NCCN guidelines insights: breast cancer, version 1.2017. J Natl Compr Canc Netw. 2017;15:433-451. [PubMed: 28404755]

20. Stuckey A, Febbraro T, Laprise J, Wilbur JS, Lopes V, Robison K. Adherence patterns to National Comprehensive Cancer Network guidelines for referral of women with breast cancer to genetics professionals. Am J Clin Oncol. 2016;39:363-367. [PubMed: 24710121]

21. Hampel H, Bennett RL, Buchanan A, Pearlman R, Wiesner GL. A practice guideline from the American College of Medical Genetics and Genomics and the National Society of Genetic Counselors: referral indications for cancer predisposition assessment. Genet Med. 2015;17:70-87. [PubMed: 25394175]

22. Halbert CH, Kessler L, Collier A, et al. Low rates of African American participation in genetic counseling and testing for BRCA1/2 mutations: racial disparities or just a difference? J Genet Couns. 2012;21:676-683. [PubMed: 22790832]

23. McCarthy AM, Bristol M, Domchek SM, et al. Health care segregation, physician recommendation, and racial disparities in BRCA1/2 testing among women with breast cancer. J Clin Oncol. 2016;34:2610-2618. [PubMed: 27161971]

24. Hayden S, Mange S, Duquette D, Petrucelli N, Raymond VM. BRCA Clinical Network Partners. Large, prospective analysis of the reasons patients do not pursue BRCA genetic testing following genetic counseling. J Genet Couns. 2017;26:859-865. [PubMed: 28093663] 
25. Han X, Jemal A. Recent patterns in genetic testing for breast and ovarian cancer risk in the U.S. Am J Prev Med. 2017;53:504-507. [PubMed: 28669566]

26. Green RF, Ari M, Kolor K, et al. Evaluating the role of public health in implementation of genomics-related recommendations: a case study of hereditary cancers using the CDC Science Impact Framework. Genet Med. 2019;21:28-37. [PubMed: 29907802] 


\section{롤 \\ 길}

ग

政

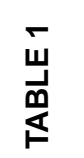

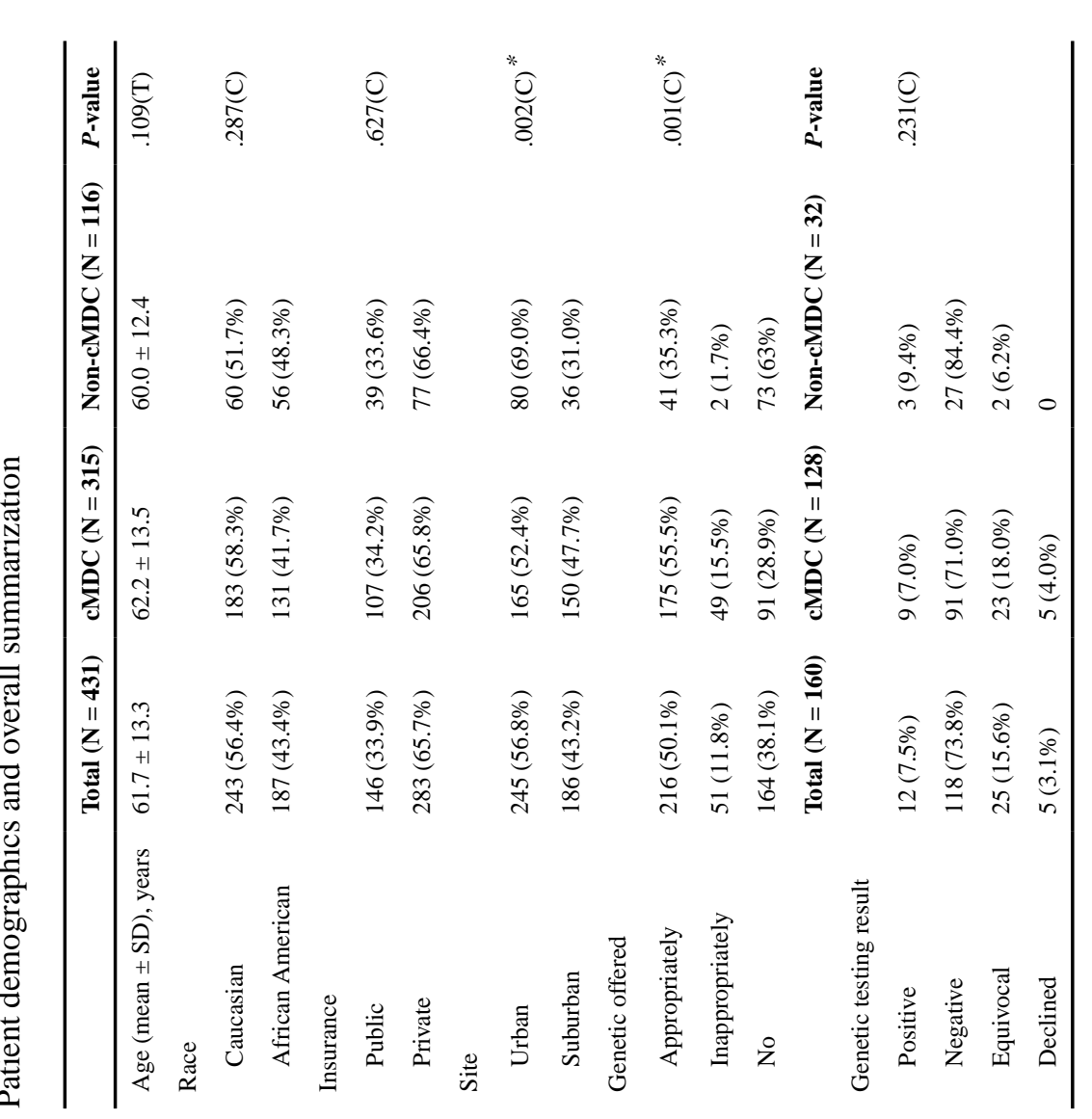

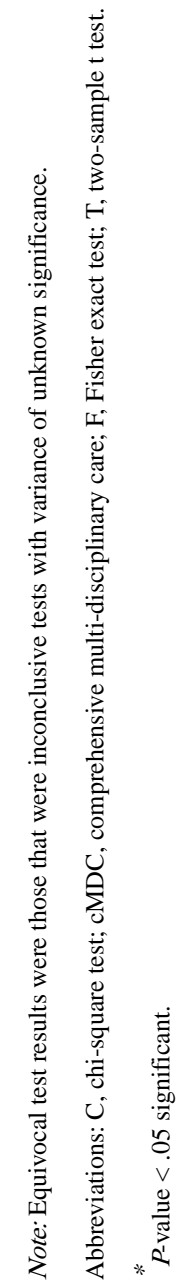

Breast J. Author manuscript; available in PMC 2020 May 24. 


\section{롤 \\ 길}

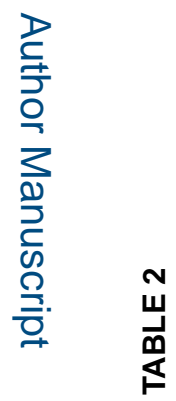

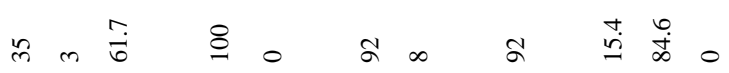

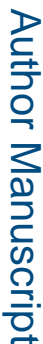

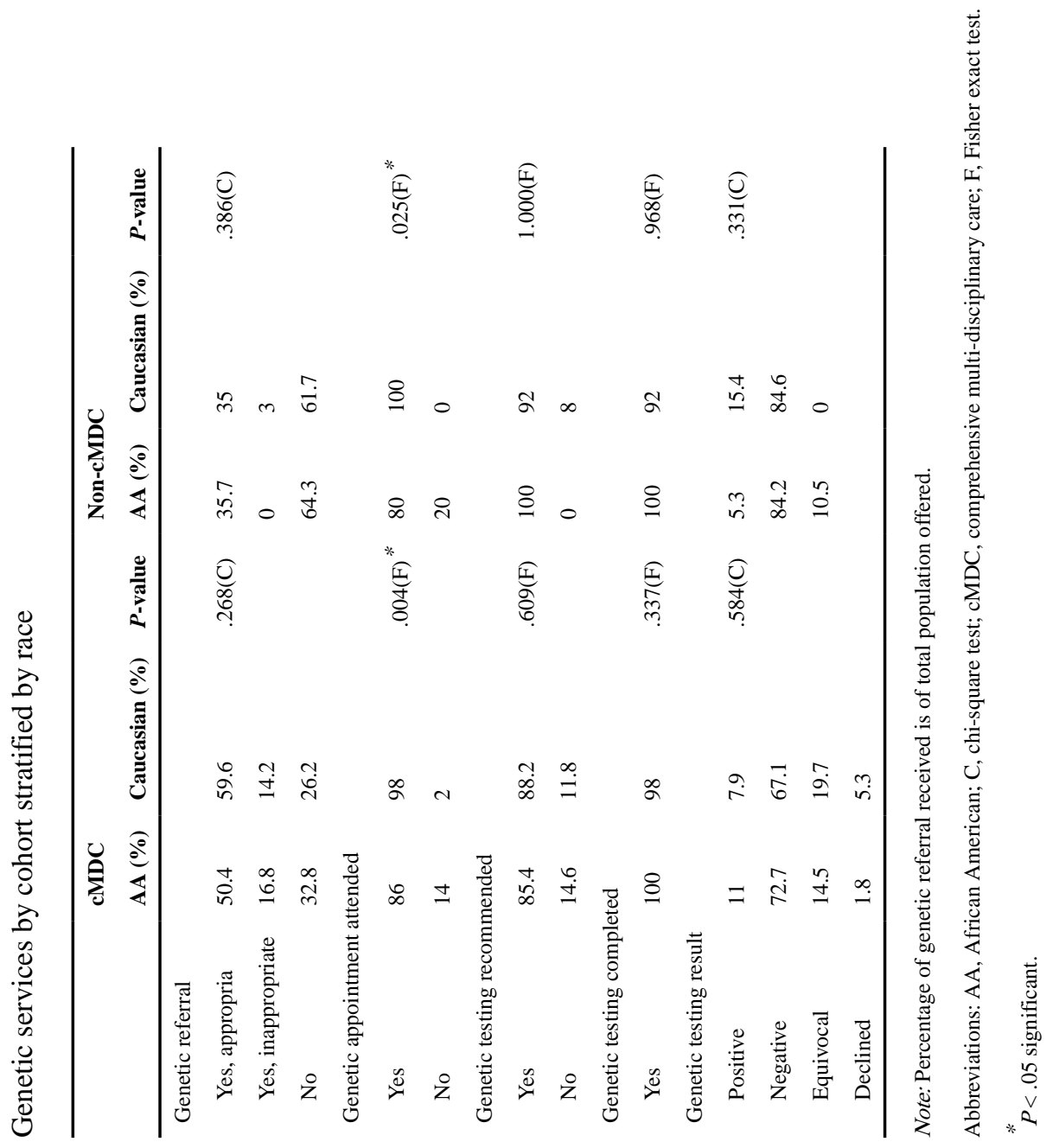

Breast J. Author manuscript; available in PMC 2020 May 24. 


\section{롤 \\ ํㅗㄹ}

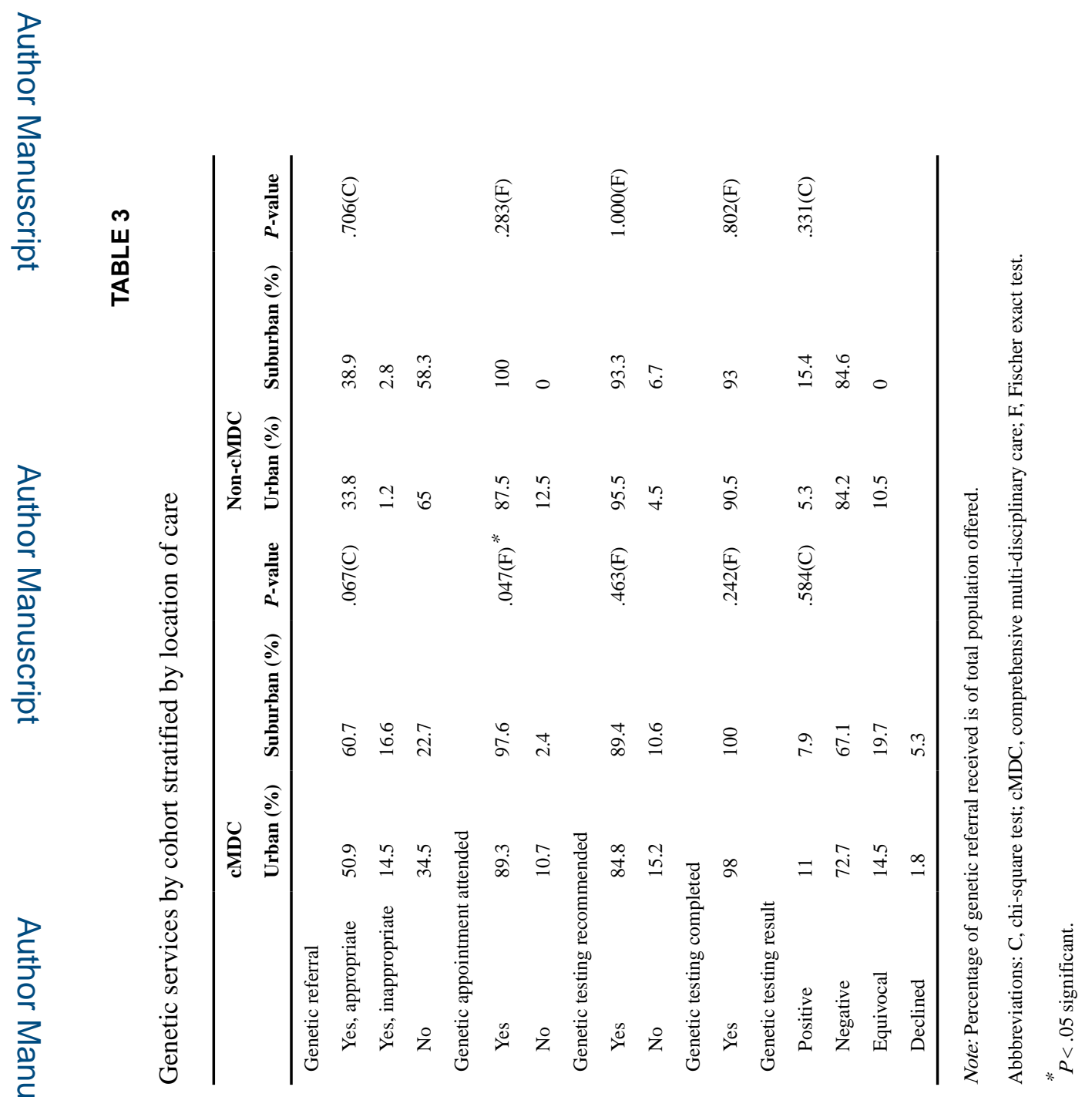

Breast J. Author manuscript; available in PMC 2020 May 24. 


\section{롤 \\ 골}

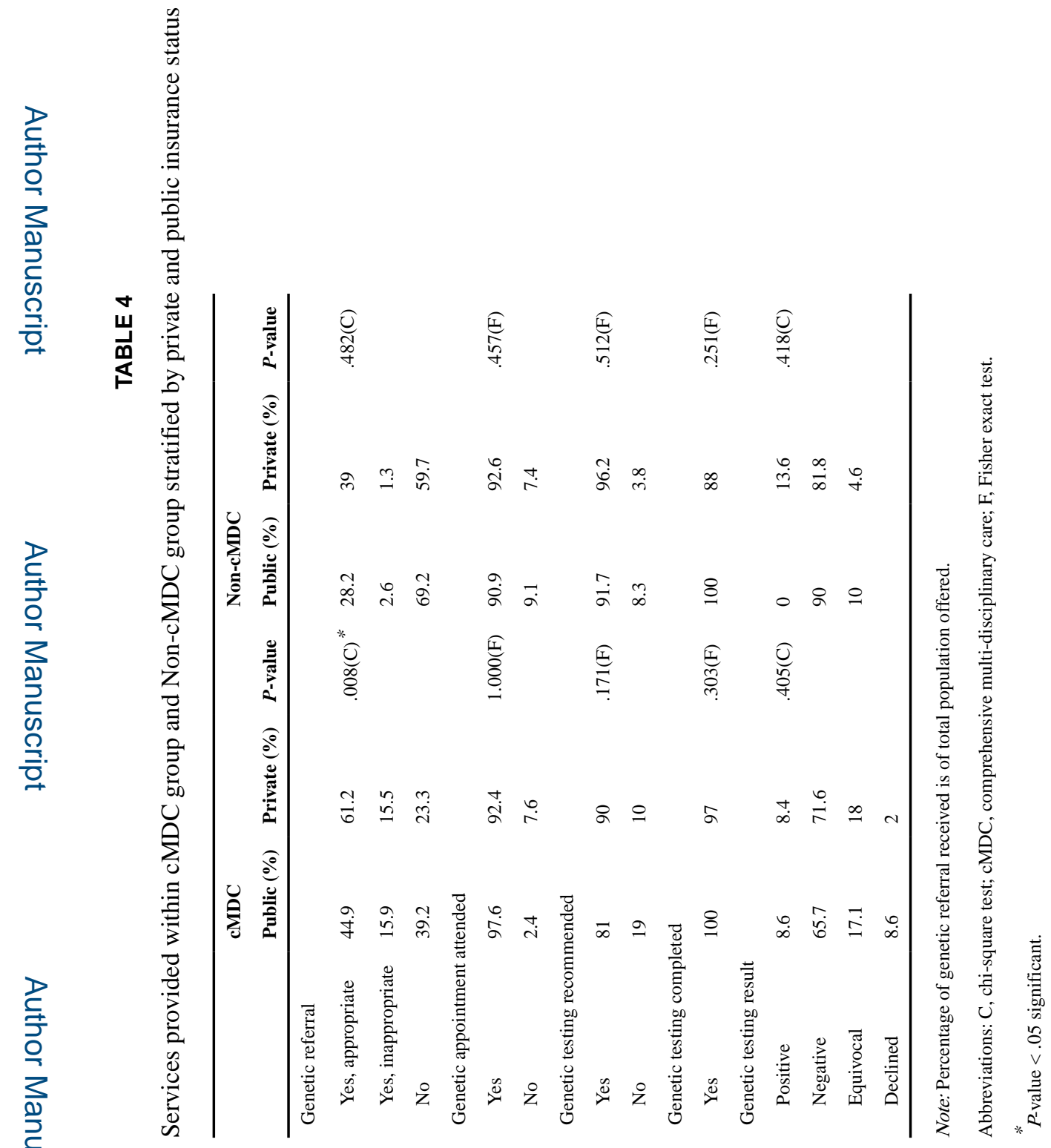

Breast J. Author manuscript; available in PMC 2020 May 24. 